\title{
Can tumour location predict degree of malignancy in small renal masses?
}

\author{
Michael A.S. Jewett, MD, FRCSC, FACS
}

See related article on page 414.

Can Urol Assoc J 2010;4(6):418

S mall renal mass (SRM), defined as $<4 \mathrm{~cm}$ in diameter, is a recognized clinical entity that is usually discovered incidentally on imaging. It is now well-known that not all SRMs are renal cell carcinoma (RCC) and that the risk of progression is difficult to predict. It is generally assumed that about $20 \%$ of SRMs are benign. Where possible, partial nephrectomy is now the preferred surgical treatment, so if the tumour turns out to be benign, the remainder of the kidney is preserved. However, there is significant morbidity from partial nephrectomy; the literature demonstrates that up to $20 \%$ of patients require transfusion, experience urinary fistula or suffer secondary bleeds. We currently believe that most benign SRMs do not require treatment, at least without evidence of growth or complications, such as bleeding or pain. How can we avoid unnecessary surgery for these benign tumours?

Imaging characteristics are not reliable predictors of malignancy. Tumour size correlates with the risk of malignancy, but this correlation is weak for smaller SRMs. The only method we currently have is biopsy. It is now recognized that needle core biopsy is safe and accurate when used as a diagnostic tool. However, the role of biopsy remains controversial and diagnostic rates vary with experience and tumour characteristics. As a result, biopsy is not yet the standard practice. If we had reliable prognostic indicators, we might avoid biopsy in some patients and go directly to treatment, confident of malignancy.

This report that tumour location may correlate with the risk of malignancy is provocative and potentially hypothesis generating if validated. ${ }^{1}$ The authors conclude that the risk of malignancy is 3.5 times higher for centrally located tumours. Why should this be? There may be methodological explana- tions that would invalidate the conclusion. Characterizing a tumour as central or peripheral is somewhat subjective, so that this retrospective analysis may have unrecognized bias. Are there biological reasons? We hypothesize that RCC arises from tubular cells or stem cells and these cells of origin may be more centrally located. This is intriguing and should be investigated further if this observation is validated. It would have implications not only for diagnosis but perhaps for therapy.

We are used to characterizing tumour location as we plan partial nephrectomy or consider thermal ablation. It would be relatively easy to codify the location prospectively to validate these findings. Even better would be the use of image processing to automatically define tumour size and location relative to the remainder of the kidney; we would then correlate these objectively defined characteristics with the pathology. Hopefully, we will see further study of these intriguing observations.

Farquharson Clinical Research Chair in Oncology, Division of Urology and Department of Surgical Oncology, Princess Margaret Hospital, University Health Network, Department of Surgery (Urology), University of Toronto, Toronto, ON

Competing interests: None declared.

This paper has been peer-reviewed.

\section{Reference}

1. Mason RJ, Abdolell M, Rendon RA. Tumour location as a predictor of benign disease in the management of renal masses. Can Urol Assoc J 2010;6:414-7.

Correspondence: Dr. Michael A.S. Jewett, Princess Margaret Hospital, 3rd Floor Rm 130, 610 University Ave, Toronto, ON M5G 2M9; m.jewett@utoronto.ca 